\title{
Distonia aguda relacionada ao uso de bromoprida em pacientes pediátricos
}

\author{
Acute dystonia after use of bromopride in pediatric patients
}

\author{
Eliane Roseli Barreira ${ }^{1}$, Ricardo Borges Magaldi²
}

\section{RESUMO}

Objetivo: Descrever dois casos de distonia aguda após uso de bromoprida em crianças e realizar revisão da literatura em relação aos mecanismos fisiopatológicos de indução de liberação extrapiramidal, sua sintomatologia e tratamento.

Descrição do caso: Caso 1: adolescente de 13 anos com quadro de dor e hipertonia cervical associados a febre, náuseas e vômitos, com hipótese inicial de meningite. A investigação subsequente revelou que o quadro iniciou-se após ingestão de uma única dose de bromoprida. $\mathrm{O}$ paciente apresentou boa resposta ao tratamento com difenidramina, sem necessidade de coleta de líquor. Caso 2: Lactente de seis meses que desenvolveu sintomas graves de liberação extrapiramidal relacionados à superdosagem de bromoprida, com reversão rápida dos sintomas após administração de biperideno.

Cometários: Este é o primeiro relato de distonia aguda após uso de bromoprida em crianças. Embora muito utilizada no Brasil como agente pró-cinético e antiemético, nenhum estudo clínico até o momento demonstrou melhor perfil de segurança da bromoprida em relação aos demais antieméticos antagonistas da dopamina. Até que tais estudos sejam realizados, sugere-se cautela na prescrição de bromoprida. Medidas não-farmacológicas devem ser recomendadas no tratamento de vômitos e da doença do refluxo gastresofágico. Quando o tratamento farmacológico for indispensável, deve-se dar preferência a drogas com perfil de segurança mais bem estabelecido.

Palavras-chave: transtornos motores; discinesia induzida por drogas; antagonistas da dopamina; antieméticos; vômito; envenenamento; antídotos.

\section{ABSTRACT}

Objective: To report the case of two patients with acute dystonia induced by bromopride in children, followed by a review of the mechanisms of induction of movement disorders by antidopaminergic anti-emetic drugs, its clinical symptoms and treatment.

Case description: Case 1: a 13 years old teenager who developed acute hypertonia and neck pain associated to fever and vomiting, suggestive of meningitis. Further investigation revealed that symptoms were associated with the ingestion of a single dose of bromopride. The symptoms stopped after administration of diphenidramine, preventing a spinal tap. Case 2: six months old infant who developed extrapyramidal movement disorder related to bromopride overdose, with prompt resolution of symptoms after treatment with biperiden.

Comments: This seems to be the first report of acute dystonia after the use of bromopride in children. Although frequently used in Brazil as an anti-emetic and prokynetic agent, no clinical study has showed that bromopride has a better safety profile than other antidopaminergic anti-emetic drugs. While such studies are not available, caution is needed in the context of pediatric prescription of bromopride. Non pharmacological measures should be adopted in the management of vomiting and gastroesophageal reflux. If medical treatment cannot be avoided, one would rather use medications with a better established safety profile.

Key-words: movement disorders; dyskinesia, drug induced; dopamine antagonists; antiemetics; vomiting; poisoning; antidotes.
Instituição: Hospital Israelita Albert Einstein (HIAE), Unidade Avançada de Alphaville, Barueri, SP, Brasil

${ }^{1}$ Médica do Pronto Atendimento de Pediatria do HIAE, Unidade Alphaville, médica assistente da Unidade de Terapia Intensiva Pediátrica do Hospital Universitário da Universidade de São Paulo (USP), São Paulo, SP, Brasil ${ }^{2}$ Gerente médico do HIAE, Unidade Avançada de Alphaville. Doutor em Pneumologia pela Faculdade de Medicina da USP, São Paulo, SP, Brasil
Endereço para correspondência

Eliane Roseli Barreira

Alameda Purus, 105 - Alphaville

CEP 06454-030 - Barueri/SP

E-mail: eliane@hu.usp.br

Recebido em: 30/6/08

Aprovado em: 1/10/08 


\section{Introdução}

Os antieméticos são medicamentos muito utilizados na prática pediátrica. Apesar de a Academia Americana de Pediatria não recomendar o uso de antieméticos no tratamento das gastrenterites agudas ${ }^{(1)}$, estudos mostram que 52 a $79 \%$ dos pediatras os utilizam para controle de vômitos associados às gastrenterites ${ }^{(2,3)}$. Muitos desses medicamentos apresentam também ação pró-cinética e são empregados no tratamento da doença do refluxo gastresofágico, embora sem eficácia claramente comprovada ${ }^{(4)}$.

Os antieméticos podem ser agrupados em três classes de acordo com seu local de ação: os antagonistas da dopamina, os bloqueadores de receptores de serotonina 5-HT3 e os antihistamínicos. Os antidopaminérgicos são os mais frequentemente relacionados ao desenvolvimento de efeitos adversos graves. No entanto, desde que foi suspensa a comercialização da cisaprida devido a eventos cardiovasculares graves ${ }^{(5)}$, sua utilização tem sido crescente ${ }^{(6)}$.

Agentes pró-cinéticos antidopaminérgicos incluem: bromoprida, metoclopramida, domperidona, levosulpirida, droperidol, cleboprida e proclorperazina. Todos agem por meio do bloqueio dos receptores D2 do trato gastrintestinal e do sistema nervoso central (SNC). Dentre os efeitos adversos comuns relacionados a várias dessas drogas, os sintomas de liberação extrapiramidal estão entre os de maior frequência e gravidade. Até o momento, nenhum caso de liberação extrapiramidal após uso de bromoprida foi relatado em crianças.

\section{Relato de casos}

\section{Caso 1}

Adolescente de 13 anos, sexo masculino, admitido no Pronto Atendimento com história de febre, náuseas e diarreia líquida há um dia. No dia da admissão, iniciaram-se dor cervical e contrações espasmódicas do pescoço. Ao exame de entrada, mostrava-se ansioso, desidratado, com frequência cardíaca $116 \mathrm{bpm}$, temperatura axilar de $36^{\circ} \mathrm{C}$ e pressão arterial de $110 \times 62 \mathrm{mmHg}$. Os exames laboratoriais mostraram: hemoglobina $13,8 \mathrm{~g} / \mathrm{dL}$ hematócrito $42 \%$, leucócitos 4700 (68\% neutrófilos, $20 \%$ linfócitos, $9 \%$ monócitos), plaquetas 224.000. Sódio $139 \mathrm{mEq} / \mathrm{L}$, potássio $\mathrm{mEq} / \mathrm{L}$, cálcio ionizado $1,22 \mathrm{mmol} / \mathrm{L}$, ureia $22 \mathrm{mg} / \mathrm{dL}$ e creatinina $0,7 \mathrm{mg} / \mathrm{dL}$.

Realizou-se hidratação endovenosa e solicitou-se avaliação neurológica e coleta de líquor (LCR) devido a uma suspeita de meningite. Quando questionado diretamente, durante a avaliação neurológica, o adolescente revelou ter ingerido um único comprimido de bromoprida três horas antes do início da sintomatologia. Foi contraindicada a coleta de LCR. Medicado com difeniframina, o paciente evoluiu com melhora gradativa dos sintomas e alta 18 horas após a admissão.

\section{Caso 2}

Lactente de seis meses, sexo masculino, com antecedente de prematuridade de causa não definida, idade gestacional de 32 semanas, peso de 2020g, boletim de Apgar de primeiro e quinto minutos de, respectivamente, 2 e 6 . Teve doença de membrana hialina leve, hipertensão pulmonar e persistência de canal arterial, resolvidos com seis dias de vida. O ultrassom de crânio era normal. Apresentava desenvolvimento neurológico compatível com a idade. Aos quatro meses de vida, apresentou episódio de gastrenterite tratada com hidratação oral e bromoprida, sem qualquer efeito adverso. Procurou o Pronto Atendimento com história de sialorreia, eructações e náuseas há dois dias. No dia da admissão, foi medicado pela família com bromoprida, em dose única equivalente a $0,8 \mathrm{mg} / \mathrm{kg}$ (dose máxima habitual: $0,3 \mathrm{mg} / \mathrm{kg}$ ). Duas horas após a ingestão do medicamento iniciou irritabilidade, desvio conjugado de olhar, hipertonia cervical e de membros superiores. Deu entrada em estado geral regular, pálido, frequência cardíaca a $142 \mathrm{bpm}$, temperatura de $36,8^{\circ} \mathrm{C}$ e saturação de $\mathrm{O}_{2}$ a $98 \%$. Ao exame neurológico, mostrava irritabilidade não consolável, fontanela normotensa, hipertonia cervical e de membros superiores, desvio lateral do pescoço, opistótono intermitente e desvio conjugado do olhar para cima. Recebeu biperideno por via endovenosa, na dose de $0,05 \mathrm{mg} / \mathrm{kg}$, com melhora do opistótono e da irritabilidade, porém com persistência do desvio conjugado de olhar uma hora após a medicação. Fez-se nova dose de biperideno com desaparecimento completo dos sintomas. O paciente teve alta hospitalar quatro horas após a admissão, sem recidiva da sintomatologia.

\section{Discussão}

\section{Funções dos receptores dopaminérgicos}

A dopamina é um neurotransmissor catecolaminanérgico, precursor da norepinefrina. É secretada pelos neurônios dopaminérgicos de origem simpática extrínseca e exerce ações no sistema nervoso central e periférico. Os receptores dopaminérgicos são divididos em duas classes: D1 e D2. Os receptores D1 têm localização pós-juncional e estimulam a adenilciclase, enquanto os receptores D2 têm localização pré e pós-juncional, inibem a adenilciclase e a liberação de 
acetilcolina nos terminais nervosos colinérgicos ${ }^{(7)}$. Estudos sugerem que cerca de $45 \%$ da dopamina do organismo seja proveniente de um sistema dopaminérgicos não neuronal, presente no trato gastrintestinal, baço e pâncreas ${ }^{(8)}$. A dopamina secretada pelo trato gastrintestinal não é metabolizada em norepinefrina e exerce ação local. O estímulo dos receptores D2 causa potente inibição da motilidade gastrintestinal, reduz o tônus do esfíncter esofágico inferior e a coordenação antroduodenal, estimula secreções exógenas e regula o fluxo sanguíneo para as mucosas ${ }^{(9)}$. O bloqueio desses receptores promove, portanto, efeito pró-cinético gastrintestinal.

No SNC, $80 \%$ dos neurônios dopaminérgicos encontram-se nas vias nigroestriatais. $\mathrm{O}$ restante distribui-se no núcleo hipotalâmico, área tegmental ventral, retina e bulbo olfatório. Receptores D2 estão presentes na área postrema; sua estimulação induz vômitos. Neurônios hipotalâmicos dopaminérgicos projetam-se na hipófise, onde a ativação dos receptores D2 bloqueia a secreção de prolactina. Portanto, além do efeito antiemético e procinético, o bloqueio dos receptores $\mathrm{D} 2$ pode induzir liberação extrapiramidal e hiperprolactinemia.

\section{Efeitos adversos dos agentes antidopaminérgicos}

A atividade motora é regulada pelo sistema extrapiramidal e modulada pelos neurotransmissores excitatórios colinérgicos e inibitórios dopaminérgicos. Por esse motivo, os efeitos adversos mais comuns das medicações antidopaminérgicas são os de liberação extrapiramidal. Embora mais relacionados aos neurolépticos, também ocorrem com os antieméticos, com maior número de casos relacionados à metoclopramida. Estima-se que a incidência de reações distônicas após uso de antieméticos seja de dois casos para cada 100 mil pessoas da população geral, chegando a até $25 \%$, com o uso de metoclopramida em crianças $^{(10)}$. A razão da maior suscetibilidade de crianças a efeitos adversos neurológicos ainda não está bem estabelecida, mas é provável que a maior permeabilidade da barreira hematoencefálica facilite a penetração das drogas no
SNC. História familiar de reações adversas a antieméticos se faz presente em até $6 \%$ dos indivíduos afetados.

A incidência de reações extrapiramidais varia de acordo com as diferentes drogas e depende de fatores como a constante de dissociação da droga, capacidade de penetração na barreira hematoencefálica, quantidade ingerida e tempo de uso. Bromoprida, metoclopramida e levosulpirida possuem alta constante de dissociação $(>10 \mathrm{nM})$, isto é, ligam-se fracamente aos receptores D2 e, por isso, têm pouca capacidade de induzir liberação extrapiramidal. A cleboprida e domperidona possuem baixa constante de dissociação (2nM) e ligam-se firmemente ao receptor D2, podendo rapidamente induzir reações extrapiramidais ${ }^{(9)}$. No entanto, a domperidona tem pouca penetração na barreira hematoencefálica, o que justifica sua relativa segurança em relação aos demais antieméticos ${ }^{(11)}$.

\section{Manifestações clínicas}

Dentre os efeitos adversos induzidos pelos antieméticos antidopaminérgicos, as reações distônicas destacam-se por sua frequência e gravidade. Podem ocorrer aguda ou tardiamente.

As reações distônicas agudas caracterizam-se por contrações musculares sustentadas que produzem movimentos repetitivos de torção, espasmos ou posturas anormais. As manifestações clínicas variam de acordo com o grupo muscular afetado (Tabela 1). Podem ocorrer logo após a ingestão de uma única dose, depois de vários dias de uso de doses terapêuticas ou, ainda, como manifestação de superdosagem. A maioria dos casos ocorre nas primeiras 72 horas de uso da medicação. A apresentação dos sintomas é, muitas vezes, dramática, especialmente em crianças. O diagnóstico diferencial deve ser feito com outras desordens neurológicas e sistêmicas, tais como convulsões parciais, meningite, tétano, distúrbios eletrolíticos e envenenamento por estricnina ${ }^{(12)}$. A resolução dos sintomas sem sequelas neurológicas ocorre na maioria dos casos, embora existam raros relatos de distonias

Tabela 1 - Manifestações clínicas de liberação extrapiramidal de acordo com o grupo muscular afetado

\begin{tabular}{|c|c|c|}
\hline Musculatura afetada & Manifestação & Sintomas clínicos \\
\hline Palpebral & Blefaroespasmo & Fechamento palpebral \\
\hline $\begin{array}{l}\text { Facial, laríngea, } \\
\text { mandibular e de língua }\end{array}$ & Crises bucolinguais & $\begin{array}{l}\text { Movimentos de deglutição ou mastigação repetitivos, } \\
\text { engasgos, assimetria facial, protrusão da língua, trismo }\end{array}$ \\
\hline Ocular extrínseca & Crises oculógiras & Desvio conjugado de olhar \\
\hline Laríngea e faríngea & Distonia laríngea & Disfonia, estridor, disgagia \\
\hline Cervical & Torcicolo & $\begin{array}{l}\text { Contratura cervical mantida ou espasmódica, desvio } \\
\text { lateral da cabeça }\end{array}$ \\
\hline Paravertebral & Opistótono & Hiperextensão de tronco \\
\hline
\end{tabular}


irreversíveis ${ }^{(3,13,14)}$. O desenvolvimento de sintomas extrapiramidais em doses habituais pode estar relacionado a um polimorfismo genético homozigótico, que induz à inatividade de uma subclasse da enzima hepática P450(15).

Distonias agudas já foram relatadas após o uso de todos os antidopaminérgicos, com maior número de casos relacionados à metoclopramida. Até o momento, nenhum caso de distonia associado à bromoprida havia sido publicado em crianças. No entanto, o pequeno número de estudos direcionados aos efeitos da bromoprida, tanto em adultos como em crianças, não permitem conclusões quanto à maior segurança dessa droga em comparação aos demais antieméticos antidopaminérgicos ${ }^{(16,17)}$. Um único estudo realizado com 4.182 adultos observou a ocorrência de discinesias em $0,4 \%$ dos pacientes tratados ${ }^{(18)}$. Apenas dois trabalhos estudaram a ação da bromoprida em crianças, abrangendo um total de 103 pacientes ${ }^{(19,20)}$. Nenhum caso de liberação extrapiramidal foi observado. A ausência de relatos de efeitos adversos neurológicos pode, na verdade, não refletir a real segurança da bromoprida, mas a restrição de sua comercialização em vários países da Europa e nos Estados Unidos.

O bloqueio prolongado dos receptores dopaminérgicos pode, ainda, induzir a parkinsonismo e discinesias tardias, inclusive em crianças ${ }^{(12,21)}$. Outros efeitos adversos comuns aos antidopaminérgicos são a hiperprolactinemia e suas consequências, como galactorreia, ginecomastia e amenorreia, por bloqueio do receptor D2 na hipófise. Como a hipófise situa-se fora da barreira hematoencefálica, a hiperprolactinemia pode ocorrer com qualquer pró-cinético antidopaminérgico e já foi relatada após uso de todos eles, incluindo a bromoprida ${ }^{(22)}$. Ginecomastia e galactorreia foram descritas em lactentes jovens e adolescentes com o uso de metoclopramida por tempo variável, desde três semanas até anos de uso contínuo ${ }^{(23)}$. Apesar de esses efeitos serem considerados adversos, tais medicações são, por vezes, indicadas com fins terapêuticos para promover a lactação em mulheres com hipogalactia ${ }^{(24)}$.

\section{Tratamento}

Várias opções terapêuticas podem ser utilizadas no tratamento das reações distônicas, embora nenhuma seja claramente superior à outra. Dentre as medicações mais utilizadas, estão os anticolinérgicos e os benzodiazepínicos.

Além da ação direta nas vias nigroestriatais, o bloqueio dopaminérgico causa também um desequilíbrio entre os neurotransmissores agonistas da atividade muscular (acetilcolina e ácido gamaminobutírico) e os antagonistas (dopamina), com predomínio dos primeiros. O tratamento direcionado ao bloqueio desses neurotransmissores excitatórios causa, portanto, alívio dos sintomas.

Medicações com ação anticolinérgica incluem o biperideno, prometazina e difenidramina. O bloqueio da acetilcolina restaura o equilíbrio excitatório-inibitório e promove o alívio dos sintomas em poucos minutos.

O biperideno deve ser administrado na dose de $0,05 \mathrm{mg} / \mathrm{kg}$, podendo ser repetido após meia hora. Quando administrado por via endovenosa, tem início de ação em 1,3 minutos e pico em 9,2 minutos ${ }^{(25)}$. Apesar de poder ser administrado por via intramuscular, em um estudo com 30 pacientes, o biperideno administrado por essa via não se mostrou superior ao placebo no alívio dos sintomas extrapiramidais ${ }^{(26)}$.

A difenidramina é um anti-histamínico bloqueador do receptor $\mathrm{H} 1$ com propriedades anticolinérgicas. Tem rápida penetração na barreira hematocefálica, onde, além dos efeitos anticolinérgicos, exerce bloqueio dos receptores muscarínicos, serotoninérgicos e alfa adrenérgicos. É amplamente utilizado no tratamento de distonias induzidas por antidopaminérgicos ${ }^{(27-29)}$. Estudos em animais sugerem que parte da ação antidistônica da difenidramina se dá por seu efeito anti-histamínico, embora o papel dos receptores histamínicos no desenvolvimento de reações distônicas ainda não esteja bem esclarecido ${ }^{(30)}$. Deve ser administrado na dose de $1,2 \mathrm{mg} / \mathrm{kg}$, por via endovenosa ou intramuscular profunda.

De modo semelhante aos anticolinérgicos, os inibidores do ácido gama aminobutírico, como o baclofeno, restauram o desequilíbrio entre agonistas e antagonistas da atividade muscular. São pouco usados no tratamento de distonias agudas, pois, por estarem disponíveis apenas para administração oral, seu início de ação é lento.

Os benzodiazepínicos não interferem no balanço dos neurotransmissores, apenas induzem relaxamento muscular imediato, além de sedação, o que causa alívio dos sintomas enquanto a droga é metabolizada. Em comparação à difenidramina, o diazepam promove reversão mais rápida dos sintomas, porém com maior efeito sedativo ${ }^{(31)}$.

Distonias irreversíveis, focais e segmentares, podem ser tratadas com injeção local de toxina botulínica, porém com resultados limitados ${ }^{(13,32)}$.

\section{Conclusões}

Este é o primeiro relato de liberação extrapiramidal após uso de bromoprida em crianças. Apesar de amplamente utilizada no Brasil, o perfil de segurança da bromoprida em 
crianças ainda não foi comprovado por estudos clínicos. Até que tais estudos sejam realizados, sugere-se mais critério na indicação da bromoprida, bem como dos demais antieméticos antidopaminérgicos. O uso de medidas não-farmacológicas no tratamento dos vômitos relacionados às gastrenterites, como hidratação oral e fracionamento da dieta, assim como recomendações dietéticas e posturais associadas à redução da produção ácida no tratamento do refluxo gastresofágico, pode prevenir a indicação desnecessária desses medicamentos. Quando as intervenções farmacológicas mostrarem-se indispensáveis, a utilização de medicamentos com segurança bem estabelecida, como a ondansetrona e a domperidona, pode evitar a ocorrência dos efeitos adversos graves e por vezes irreversíveis dos antidopaminérgicos.

\section{Referências bibliográficas}

1. No authors listed. Practice parameter: the management of acute gastroenteritis in young children. American Academy of Pediatrics, Provisional Committee on Quality Improvement, Subcommittee on Acute Gastroenteritis. Pediatrics 1996;97:424-35.

2. Albano F, Bruzzese E, Spagnuolo MI, De Marco G. Antiemetics for children with gastroenteritis: off-label but still on in clinical practice. J Pediatr Gastroenterol Nutr 2006;43:402-4.

3. Kwon KT, Rudkin SE, Langdorf MI. Antiemetic use in pediatric gastroenteritis: a national survey of emergency physicians, pediatricians, and pediatric emergency physicians. Clin Pediatr (Phila) 2002;41:641-52.

4. Shaffer SE, Levine S. Gastroesophageal reflux in children. Hosp Pharm 2003;38:212-7.

5. Tonini M, De Ponti F, Di Nucci A, Crema F. Review article: cardiac adverse effects of gastrointestinal prokinetics. Aliment Pharmacol Ther 1999;13:1585-91.

6. Glessner MR, Heller DA. Changes in related drug class utilization after market withdrawal of cisapride. Am J Manag Care 2002;8:243-50.

7. Willems JL, Buylaert WA, Lefebvre RA, Bogaert MG. Neuronal dopamine receptors on autonomic ganglia and sympathetic nerves and dopamine receptors in the gastrointestinal system. Pharmacol Rev 1985;37:165-216.

8. Eisenhofer G, Aeman A, Friberg P, Hooper D, Fandriks L, Lonroth $\mathrm{H}$ et al. Substantial production of dopamine in the human gastrointestinal tract. J Clin Endocrinol Metab 1997;82:3864-71.

9. Tonini M, Cipollina L, Poluzzi E, Crema F, Corazza GR, De Ponti F. Review article: clinical implications of enteric and central D2 receptor blockade by antidopaminergic gastrointestinal prokinetics. Aliment Pharmacol Ther 2004;19:379-90.

10. Montvale NJ. Metoclopramide. In: Phisicians' desk reference. $50^{\text {th }}$ ed. Montvale: Medical Economics; 1996. p. 2068-70.

11. Barone JA. Domperidone: mechanisms of action and clinical use. Hosp Pharm 1998;33:191-7.

12. Dingli K, Morgan R, Leen $\mathrm{C}$. Tetanus versus acute dystonic reaction caused by metoclopramide. BMJ 2007;334:899-900.

13. Grimes JD, Hassan MN, Preston DN. Adverse neurologic effects of metoclopramide. Can Med Assoc J 1982;126:23-5.

14. Walker M, Samii A. Chronic severe dystonia after single exposure to antiemetics. Am J Emerg Med 2006;24:125-7.

15. Van der Padt A, Van Schaik RH, Sonneveld P. Acute dystonic reaction to metoclopramide in patients carrying homozygous cytochrome P450 2D6 genetic polymorphisms. Neth J Med 2006;64:160-2.

16. Ferraù O, Pustorino S, Luzza G, Turiano S, Familiari L, Consolo F et al. Comparative study of the effects of bromopride and metoclopramide on gastric emptying in humans. Clin Ter 1980;93:33-8.
17. Ferraù O, Pustorino S, Luzza G, Turiano S, Familiari L, Fanfani A et al. Changes induced with bromopride and metoclopramide in the kinetics of gastric emptying in patients with gastric motor hyposthenia. Comparison with placebo. Clin Ter 1981;99:63-71.

18. Fischer M, Jesau R, Tinhof W. Management of gastrointestinal diseases using a motility-regulating preparation. Results of a field study using bromopride (Viaben) conducted by 530 general practitioners on 4182 patients. Fortschr Med 1979;97:883-8

19. Barbieri D, Scatigno Neto A, Koda YK, Pereira Filho OD. A ação do bromoprida sobre a motricidade antropilórica e esôfago-gástrica - estudo radiológico em crianças. Pediatria (S. Paulo) 1982;4:219-24.

20. Vialatte J. Experiência clínica em crianças com o bromopride - VAL 1308. Folha Med 1981;83:79-81.

21. Putnam PE, Orenstein SR, Wessel HB, Stowe RM. Tardive dyskinesia associated with use of metoclopramide in a child. J Pediatr 1992;121:983-5.

22. Gasparoni P, Fioretti D, Roncato F, Rota G, Conte N. Effect of bromopride on prolactin secretion. Clin Ter 1982;102:397-401.

23. Madani S, Tolia V. Gynecomastia with metoclopramide use in pediatric patients. J Clin Gastroenterol 1997;24:79-81.

24. Gabay MP. Galactogogues: medications that induce lactation. J Hum Lact 2002;18:274-9.

25. Hirose S, Ashby CR. Intravenous biperiden in akathisia: an open pilot study. Int J Psychiatry Med 2000;30:185-94.

26. Baskak B, Atbasoglu EC, Ozguven HD, Saka MC, Gogus AK. The effectiveness of intramuscular biperiden in acute akathisia: a double-blind, randomized, placebo-controlled study. J Clin Psychopharmacol 2007;27:289-94.

27. Vinson DR. Drotts DL. Diphenhydramine for the prevention of akathisia induced by prochlorperazine: a randomized, controlled trial. Ann Emerg Med 2001;37:125-31.

28. Yis U, Ozdemir D, Duman M, Unal N. Metoclopramide induced dystonia in children: two case reports. Eur J Emerg Med 2005;12:117-9.

29. Lou E, Abou-Zeid N. A case of metoclopramide-induced oculogyric crisis in a 16-year-old girl with cystic fibrosis. South Med J 2006;99:1290-1.

30. van't Groenewout JL, Stone MR, Vo VN, Truong DD, Matsumoto RR. Evidence for the involvement of histamine in the antidystonic effects of diphenhydramine. Exp Neurol 1995;134:253-60.

31. Parlak I, Erdur B, Parlak M, Ergin A, Ayrik C, Tomruk O et al. Midazolam vs. diphenhydramine for the treatment of metoclopramide-induced akathisia: a randomized controlled trial. Acad Emerg Med 2007;14:715-21.

32. Fabiani G, Teive HA, Germiniani F, Sá D, Werneck LC. Clinical and therapeutical features in 135 patients with dystonia: experience of movement disorders unity of the Hospital de Clínicas of the Universidade Federal do Paraná. Arq Neuropsiquiatr 1999;57:610-4. 